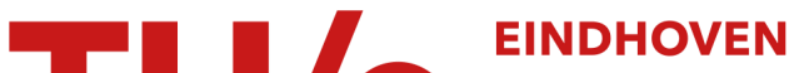 UNIVERSITY OF TECHNOLOGY
}

\section{Energy management of hybrid vehicles with state constraints}

Citation for published version (APA):

Sánchez, M., Delprat, S., \& Hofman, T. (2020). Energy management of hybrid vehicles with state constraints: a penalty and implicit Hamiltonian minimization approach. Applied Energy, 260, [114149].

https://doi.org/10.1016/j.apenergy.2019.114149

\section{Document license: \\ TAVERNE}

DOI:

10.1016/j.apenergy.2019.114149

Document status and date:

Published: 15/02/2020

\section{Document Version:}

Publisher's PDF, also known as Version of Record (includes final page, issue and volume numbers)

\section{Please check the document version of this publication:}

- A submitted manuscript is the version of the article upon submission and before peer-review. There can be important differences between the submitted version and the official published version of record. People interested in the research are advised to contact the author for the final version of the publication, or visit the $\mathrm{DOI}$ to the publisher's website.

- The final author version and the galley proof are versions of the publication after peer review.

- The final published version features the final layout of the paper including the volume, issue and page numbers.

Link to publication

\section{General rights}

Copyright and moral rights for the publications made accessible in the public portal are retained by the authors and/or other copyright owners and it is a condition of accessing publications that users recognise and abide by the legal requirements associated with these rights.

- Users may download and print one copy of any publication from the public portal for the purpose of private study or research.

- You may not further distribute the material or use it for any profit-making activity or commercial gain

- You may freely distribute the URL identifying the publication in the public portal.

If the publication is distributed under the terms of Article $25 f a$ of the Dutch Copyright Act, indicated by the "Taverne" license above, please follow below link for the End User Agreement:

www.tue.nl/taverne

Take down policy

If you believe that this document breaches copyright please contact us at:

openaccess@tue.nl

providing details and we will investigate your claim. 


\title{
Energy management of hybrid vehicles with state constraints: A penalty and implicit Hamiltonian minimization approach
}

\author{
Marcelino Sánchez ${ }^{\mathrm{a}, *}$, Sébastien Delprat ${ }^{\mathrm{a}}$, Theo Hofman ${ }^{\mathrm{b}}$ \\ ${ }^{\text {a } U n i v . ~ P o l y t e c h n i q u e ~ H a u t s-d e-F r a n c e, ~ U M R ~} 8201$ - LAMIH, F-59313 Valenciennes, France \\ ${ }^{\mathrm{b}}$ Eindhoven University of Technology, Department of Mechanical Engineering, Eindhoven $5600 \mathrm{MB}$, the Netherlands
}

\section{H I G H L I G H T S}

- Presents a novel implicit Hamiltonian minimization approach with penalty functions.

- It solves the multivariable energy management with mixed input-state constraints.

- The approach shows to be up to 46 times faster than dynamic programming.

\section{A R T I C L E I N F O}

\section{Keywords:}

Energy management

Hybrid electric vehicles

Pontryagin's minimum principle

Mixed input-state constraints

Penalty function approach

\begin{abstract}
A B S T R A C T
When designing hybrid vehicles, the energy management is formulated as an optimal control problem. The Pontryagin's minimum principle represents a powerful methodology capable of solving the energy management offline. Moreover, the Pontryagin's minimum principle has been proved useful in the derivation of online energy management algorithms, such as the equivalent consumption minimization strategy. Nevertheless, difficulties on the application of the Pontryagin's minimum principle arise when state constraints are included in the definition of the problem. A possible solution is to combine the Pontryagin's minimum principle with a penalty function approach. This is done by adding functions to the Hamiltonian, which increase the value of the Hamiltonian whenever the optimal trajectory violates its constraints. However, the addition of penalty functions to the Hamiltonian makes it harder to compute its minimum. This work proposes an effective penalty approach through an implicit Hamiltonian minimization. The proposed method is applied to solve the energy management for a hybrid electric vehicle modeled as a mixed input-state constrained optimal control problem with two states: the battery temperature and state-of-energy. It is demonstrated to be up to 46 times faster than the dynamic programming method while taking benefits of state-of-the-art boundary value problem solvers and avoiding any issue related to state quantization.
\end{abstract}

\section{Introduction}

Hybrid electric vehicles (HEV) are one of the approaches aimed to reduce the dependency on finite fossil fuel resources used for transportation. Within an HEV, the power demand from the driver is split among the different powertrain energy sources in order to operate the internal combustion engine (ICE) in its most efficient region available, thus reducing the fuel consumption. The algorithm applied to achieve the best power split is known as energy management strategy (EMS) [1].

Although EMS mostly focusses on fuel efficiency, additional criteria may be considered as well, such as reducing the emission of pollutants $[2,3]$, improving the driver's comfort [4,5], and prolonging the lifetime of the energy sources [6,7]. Real-time EMS [8] are used to operate actual cars and can only rely on causal algorithms. In simulation, finding the offline-EMS consists in computing (eventually, in a noncausal way) the ultimate performance of the HEV. In practice, the obtained results allow assessing, from an energy consumption perspective, different vehicle architectures and component sizing [9]. They can also be used to evaluate the performance of real-time energy management algorithms.

EMS in its most general form can be formulated as a mixed inputstate constrained optimal control problem with discrete and continuous inputs and dynamics. The methods for solving this optimal control problem can be classified in three groups: dynamic programming (DP), direct-approach methods, and indirect-approach methods. The

\footnotetext{
* Corresponding author.

E-mail addresses: Marcelino.SanchezPantoja@uphf.fr (M. Sánchez), Sebastien.Delprat@uphf.fr (S. Delprat), t.hofman@tue.nl (T. Hofman).
} 

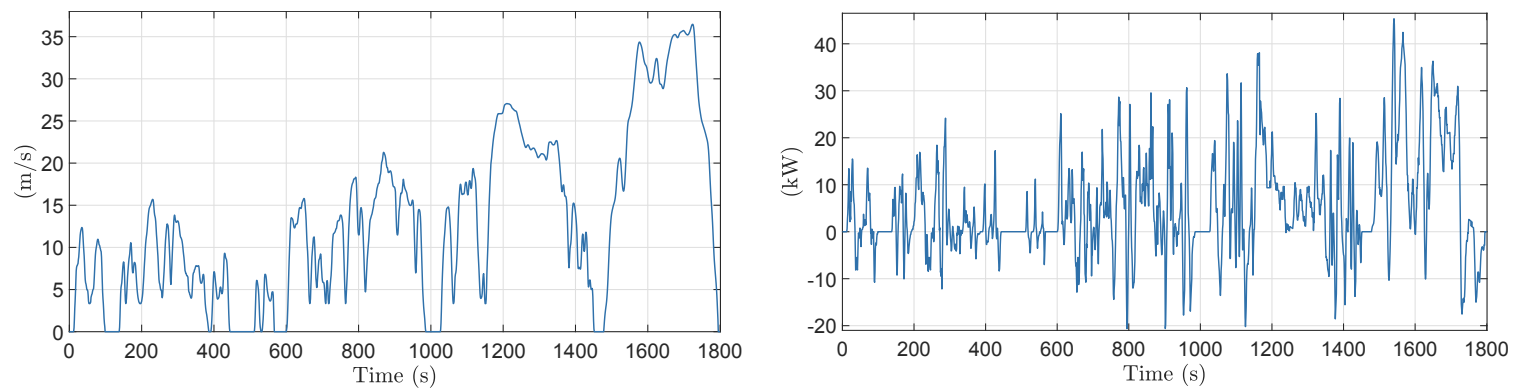

Fig. 1. Worldwide Light-vehicle Test Cycle Class 3 (WLTC-C3) in m/s (left). Power demand signal $w(t)$ for the WLTC-C3 (right).

contribution of this work lies within the third group, the indirectmethods. However, a general discussion on the three groups of methods will be provided before entering into the details of the contribution.

In the DP approach, all the variables, inputs, states and time, are quantized. Then, the resulting discrete static optimization problem is solved to determine the optimal EMS. The DP approach is widely used in the literature, since it can solve the EMS in its most general form, i.e., with hybrid dynamics and input and state constraints. The main drawback of this method comes from its computational expensiveness, referred to as the curse of dimensionality [10]. Namely, the number of elementary operations has an exponential relationship with respect to the number of states and inputs in the problem formulation. Likewise, the memory requirements increase exponentially with respect to the number of states. As a consequence, DP is, usually, restricted to problems with only one $[11,12]$, or two continuous states $[13,14]$.

In the direct-approach methods, only the time is often quantized (finite-time problems). The resulting static minimization problem is solved using mathematical programming techniques such as nonlinear programming, e.g., sequential quadratic programming (SQP) $[15,16]$, or convex programming, e.g., second-order cone programming (SOCP) [17], quadratic programming (QP) [18], and linear programming (LP) $[19,20]$. State constraints can be taken into account. Optimizing discrete controls leads to very large mixed-integer programming problems due to the considered long optimization horizon (typically, more than 1000 time steps). These problems are not tractable in general, even if they are restricted to be mixed-integer linear programming (MILP) problems. Nonetheless, when only continuous controls are considered, the direct approach does not posses the curse of dimensionality suffered by DP, therefore, it can be applied to obtain the optimal EMS, when several continuous states are considered. Moreover, it has been even applied to obtain, simultaneously, the optimal EMS and the optimal sizing of some components of the powertrain [21].

In the group of indirect methods, the calculus of variations or the Pontryagin minimum principle (PMP) is applied to obtain optimality conditions. The original EMS is then reduced to a simpler equivalent problem, namely, a boundary value problem (BVP). Hybrid-PMP $[22,23]$ allows considering both continuous and discrete systems, and thus, handling discrete controls signals, although they can lead to singular control issues [24]. State constraints lead to many theoretical difficulties, and there is no algorithm available to efficiently derive a solution in the general case. An algorithm is proposed in [25] to solve the EMS problem restricted to a single state.

Problems with many constrained states can be handled using the penalty function approach. It consists in adding an additional cost to the criterion that enforces the state to stay in the feasible region. This approach converts the state constrained problem into an unconstrained one that can be solved using the classical PMP [26,27]. Nevertheless, the resulting optimality conditions are significantly more difficult to solve [28].

The contribution of this work is a novel method for the minimization of the Hamiltonian that overcomes the difficulties of the penalty approaches to satisfy the optimality conditions. The novel method is based on an implicit Hamiltonian minimization that, under strict convexity assumptions, can solve the EMS formulated with $n$ states and $m$ inputs under mixed input-state constraints. Although other penalty approaches have been successfully applied to the EMS under state constraints, they have been so far restricted to formulations with only one state $[26,27]$. Increasing the number of states and inputs is relevant since it is necessary to produce a more accurate representation of the vehicle and all its subsystems that have an effect on the energy management [29]. Preliminary results of this work were presented in [28].

The the paper is organized as follows: Section 2 presents the vehicle modeling and the EMS formulation as an optimal control problem. The main results, an indirect-approach method based on penalty functions and an implicit Hamiltonian minimization, are presented in Section 3. Section 4 introduces algorithms to overcome numerical difficulties of the indirect-approach method. In Section 5, the indirect-approach method is applied to compute the EMS formulated in Section 2. At last, Section 6 contains the conclusions and some perspectives on possible future work.

\section{Problem statement}

The hybrid vehicle EMS consists in computing the optimal power split between the different energy sources, when the vehicle is following a prescribed velocity profile, also known as driving cycle. An example of a driving cycle, the Worldwide Light-vehicle Test Cycle Class 3 (WLTC-C3), is depicted in Fig. 1 (left). For the considered series hybrid vehicle, see Fig. 2, the EMS input data is the power $w(t)(\mathrm{kW})$ required to propel the vehicle along the given driving cycle [1].

In the rest of this section, the offline-EMS will be defined in more detail for the considered series hybrid vehicle. However, it is worth noticing that the methodology presented in this work can be applied to many other hybrid vehicle topologies as well.

\subsection{Series-HEV}

Consider the HEV powertrain topology displayed in Fig. 2. The power required for propulsion at the input of the traction motor (TM), $w(t) \in \mathbb{R}$, must be provided at each instant by the auxiliary power unit (APU), $u(t) \in \mathbb{R}$, and/or the battery pack, $P_{b}(t) \in \mathbb{R}$. Moreover, the

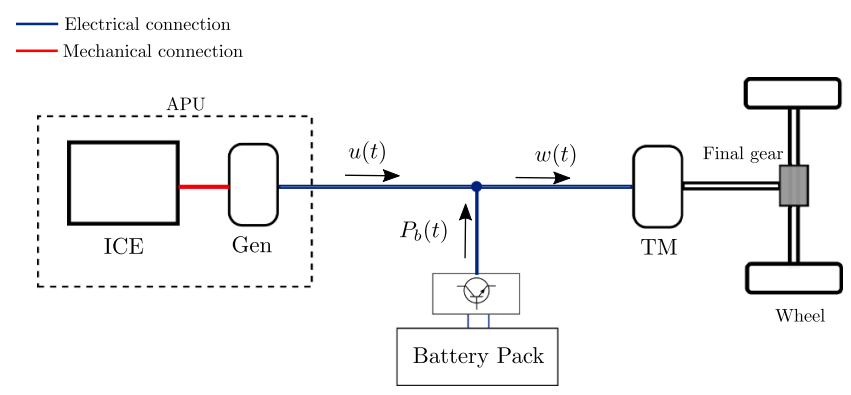

Fig. 2. Diagram of the series-HEV powertrain. 
APU is subject to a on/off command signal, $\vartheta(t) \in\{0,1\}$. The series topology imposes the following relationship among the power signals:

$w(t)=\vartheta(t) \cdot u(t)+P_{b}(t)$.

The models required to compute $w(t), P_{b}(t)$, and $u(t)$ are discussed in the following subsections. For the ease of readiness, in the following, the time dependence of the variables will be omitted when convenient.

\subsubsection{Traction subsystem}

The traction subsystem includes the vehicle model and transmission from the wheels to the TM. The corresponding model allows evaluating the energetic requirement of the vehicle. Given a velocity profile $v(t)$ and the vehicle parameters, the torque $T_{w}(t)$ and the angular velocity at the wheels $\omega_{w}(t)$, required to follow the driving cycle, are computed [1]:

$T_{w}(t)=r_{w}\left(m_{e q} \cdot \frac{\mathrm{d} v(t)}{\mathrm{d} t}+m \cdot g \cdot c_{r}+\frac{1}{2} \rho_{a} \cdot A_{f} \cdot c_{d} \cdot v^{2}(t)\right)$,

$\omega_{w}(t)=v(t) / r_{w}$,

with $c_{r}$ the tire rolling resistance $(-), g$ the gravity acceleration $\left(\mathrm{kg} \cdot \mathrm{m} / \mathrm{s}^{2}\right), \rho_{a}$ the air density $\left(\mathrm{kg} / \mathrm{m}^{3}\right), A_{f}$ the vehicle frontal area $\left(\mathrm{m}^{2}\right), c_{d}$ the drag coefficient $(-), \gamma$ the final gear ratio $(-), r_{w}$ the radius of the wheels $(\mathrm{m}), m$ the vehicle mass $(\mathrm{kg})$, and $m_{e q}=m+J_{t m} /\left(\gamma^{2} r_{w}^{2}\right)$ the equivalent mass of the vehicle $(\mathrm{kg})$. The vehicle is rear wheel driven (RWD): when braking, $T_{w}<0$, only $40 \%$ of the torque is assumed to be provided by regenerative braking and the remaining $60 \%$ is assumed to be provided by the mechanical brakes. Indicated with $\mu_{b}(t)$ as a piecewise constant torque brake factor. A constant torque brake factor for regenerative braking is considered for the sake of simplicity. More advanced blending braking strategies may be applied, as in [30]. Finally, the TM torque and speed, denoted as $T_{t m}$ and $\omega_{t m}$, are derived:

$T_{t m}(t)=\gamma \cdot \mu_{b}(t) \cdot T_{w}(t)$,

$\mu_{b}(t)=\left\{\begin{array}{cc}0.4, & T_{w}(t)<0 \\ 1, & \text { otherwise }\end{array}\right.$

$\omega_{t m}(t)=\omega_{w}(t) / \gamma$.

At last, the power demand signal $w(t)$ is computed using the traction motor efficiency map $\eta_{t m}$, given in Appendix C:

$w(t)=T_{t m}(t) \omega_{t m}(t) \eta_{t m}\left(T_{t m}(t), \omega_{t m}(t)\right)^{-s i g n}\left(T_{t m}(t)\right)$

Given the WLTC-C3 driving cycle, the power demand signal $w(t)$ is displayed in Fig. 1 (right).

\subsubsection{Battery pack}

The battery pack is connected to a DC/DC converter that fixes the DC bus voltage. Its losses are assumed to be negligible. The energetic behavior is modeled using both the state-of-energy dynamics given in [18] and a thermal model similar to the one found in [31]. They relate the battery power, $P_{b}(t)$, to the state-of-energy, denoted as $x_{1}(t)$, and the battery temperature, denoted as $x_{2}(t)$. The battery state-of-energy, $x_{1}(t)$, is derived from an equivalent electric circuit model. It consists of an open circuit voltage $U\left(x_{1}(t)\right)$ in series with a temperature dependent resistance $R_{b}\left(x_{2}(t)\right)$. The state-of-energy, $x_{1}(t)$, has the following dynamics:

$\dot{x}_{1}(t)=\frac{1}{\bar{U} \cdot Q}\left[-P_{b}(t)-\frac{R_{b}\left(x_{2}(t)\right)}{U^{2}\left(x_{1}(t)\right)} P_{b}^{2}(t)\right]$,

with $\bar{U}$ the maximum open circuit voltage (V) and $Q$ the charge capacity of the battery pack (A.s). The battery state-of-energy, $x_{1}(t)$, is constrained between $\underline{x}_{1}$ and $\bar{x}_{1}{ }^{1}$

\footnotetext{
${ }^{1}$ Throughout the paper, an underline (_) and an overline ( $\left(^{-}\right)$are used to denote minimum and maximum value, respectively.
}

$\underline{x}_{1} \leqslant x_{1}(t) \leqslant \bar{x}_{1}$.

Within the battery pack operating range (30\%-90\%), $U$ is modeled as a linear function of $x_{1}: U\left(x_{1}\right)=a_{x_{1}}+b_{x_{1}} x_{1}$, derived from $\mathrm{LiFePO}_{4}$ battery cell data found in [32], as shown in Fig. 3. The resistance $R_{b}(\Omega)$ is given by a linear function of $x_{2}(\mathrm{~K})$.

The battery pack power $P_{b}(t)$ is constrained by the current limitations of the battery pack:

$\underline{P}_{b}\left(R_{b}, U\right) \leqslant P_{b}(t) \leqslant \bar{P}_{b}\left(R_{b}, U\right)$,

with $\underline{P}_{b}=\underline{I} \cdot U-R_{b} \cdot \underline{I}^{2}$ and $\bar{P}_{b}=\bar{I} \cdot U-R_{b} \cdot \bar{I}^{2}, \underline{I}$ the minimum, and $\bar{I}$ the maximum battery pack current (A).

The dynamics of $x_{2}$ is obtained from a thermal balance energy equation $[18,31,33,34]$ :

$\dot{x}_{2}(t)=\frac{1}{C_{b}}\left[h \cdot\left(T_{\infty}-x_{2}(t)\right)+\frac{R_{b}\left(x_{2}(t)\right)}{U^{2}\left(x_{1}(t)\right)} P_{b}^{2}(t)\right]$

with $C_{b}$ the heat capacity of the battery pack $(\mathrm{kJ} / \mathrm{K}), T_{\infty}$ the ambient temperature (K), and $h$ the heat transfer coefficient between the battery pack and its surroundings $(\mathrm{W} / \mathrm{K})$. The term depending on $P_{b}$ stands for the heat dissipated by the battery resistance $R_{b}$. The battery resistance increases for low temperatures, thus reducing both the battery pack efficiency and the maximum power $\bar{P}_{b}$.

\subsubsection{Auxiliary power unit}

The APU consists of one ICE coupled with one generator (Gen) to produce electrical energy using fuel. The APU instantaneous fuel consumption, $\dot{m}_{f}(t)(\mathrm{g} / \mathrm{s})$, required to generate a given electric power $u(t)$ (W), is estimated by the following quadratic function $[18,35]$ :

$\dot{m}_{f}(u(t))=a+b \cdot u(t)+c \cdot u^{2}(t)$.

The data used to compute (12) is given in Appendix C. The criterion to be minimized by the EMS is the total fuel consumption:

$J=\int_{0}^{t_{f}}\left[\dot{m}_{f}(u(t)) \vartheta(t)+d \cdot(1-\vartheta(t)) u^{2}(t)\right] \mathrm{d} t$,

where $t_{f}$ stands for the duration of the driving cycle and $d$ is a conversion factor $\left(\frac{\mathrm{g}}{(\mathrm{kW})^{2} \cdot \mathrm{s}}\right)$. The additional term $(1-\vartheta) \cdot u^{2}$ allows enforcing $u=0$, whenever $\vartheta=0$ [28].

Optimizing the binary variable $\vartheta$ may lead to theoretical difficulties, such as singular controls [24] and non-unique optimal solutions. In order to keep the analysis simple enough and focus on the state constraints handling, the binary signal $\vartheta$ is assumed to be fixed beforehand. For instance, it can be computed using a set of empirical rules according to the power demand $w(t)$. The power produced by the APU is constrained by the physical limits of its components:

$0 \leqslant u(t) \leqslant \bar{u}$,

yet also by other components of the powertrain via (1):

$w(t)-\bar{P}_{b}(t) \leqslant u(t) \leqslant w(t)-\underline{P}_{b}(t)$.

Constraints (14) and (15) can be combined together:

$\underline{u}^{\prime}(t) \leqslant u(t) \leqslant \bar{u}^{\prime}(t)$,

$\underline{u}^{\prime}(t)=\max \left[0, w(t)-\bar{P}_{b}\right]$,

$\bar{u}^{\prime}(t)=\min \left[\bar{u}, w(t)-\underline{P}_{b}\right]$.

\subsection{Energy management as an optimal control problem}

Over a known driving cycle, the energy management problem can be formulated as an optimal control problem (OCP) in the following way:

$\min J(u)=\int_{0}^{t_{f}}\left[\vartheta \cdot\left(a+b \cdot u+c \cdot u^{2}\right)+d \cdot(1-\vartheta) \cdot u^{2}\right] \mathrm{d} t$, 

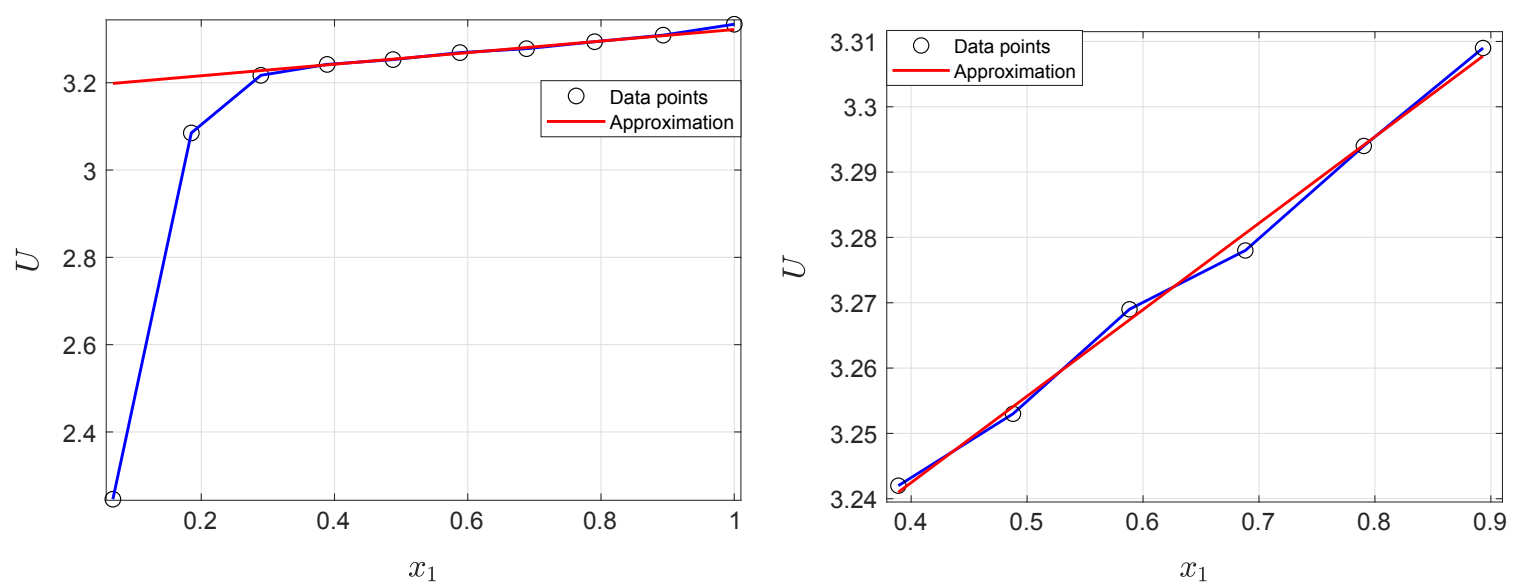

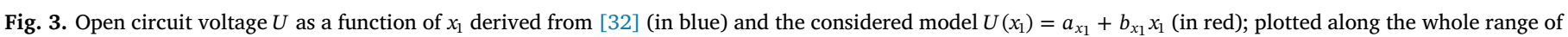
available data (left); plotted along the operating range (right). The parameters of $U\left(x_{1}\right)$ are $a_{x_{1}}=27.14$ and $b \cdot x_{1}=653.85$.

subject to:

$$
\begin{aligned}
& \dot{x}_{1}(t)=\frac{1}{\bar{U} \cdot Q}\left[-(w-\vartheta u)-\frac{R_{b}\left(x_{2}\right)}{U^{2}\left(x_{1}\right)}(w-\vartheta u)^{2}\right], \\
& \dot{x}_{2}(t)=\frac{1}{C_{b}}\left[h \cdot\left(T_{\infty}-x_{2}\right)+\frac{R_{b}\left(x_{2}\right)}{U^{2}\left(x_{1}\right)}(w-\vartheta u)^{2}\right], \\
& \underline{u}^{\prime}(t) \leqslant u(t) \leqslant \bar{u}^{\prime}(t), \\
& \underline{u}^{\prime}(t)=\max \left[0, w(t)-\bar{P}_{b}(t)\right] \\
& \bar{u}^{\prime}(t)=\min \left[\bar{u}, w(t)-\underline{P}_{b}(t)\right] . \\
& \underline{x}_{1} \leqslant x_{1}(t) \leqslant \bar{x}, \\
& x_{1}(0)=x_{1,0} \\
& x_{2}(0)=x_{2,0} \\
& x_{1}\left(t_{f}\right)=x_{1, f} .
\end{aligned}
$$

The final condition $x_{1}\left(t_{f}\right)$ allows to guarantee that a certain amount of energy will remain in the battery at the end of the driving cycle. The final state-of-energy $x_{1}\left(t_{f}\right)$ is often assumed to be equal to $x_{1}(0)$ to allow a fair comparison with respect to the fuel consumption of conventional vehicles.

The problem statement above is a state and input constrained OCP formulation for the EMS. As it accounts for the battery thermal modeling, its solutions allows investigating the effect that a battery operating under low-temperature has on the fuel consumption.

\section{Penalty function approach and implicit Hamiltonian minimization approach}

This first part of this section explains how (17) can be solved through an equivalent unconstrained OCP by means of the penalty function approach. The second part explains how the PMP optimality conditions can transform the OCP formulation into an equivalent BVP. The optimal control must be chosen to minimize the Hamiltonian, a given scalar function. Due to the considered penalties, this minimization cannot be carried out explicitly. Hence, the last subsection focuses on an implicit minimization scheme.

\subsection{Penalty function approach}

In the penalty approach, inequality constraints (17d) and (17e) are taken into account by extra terms, denoted as penalties, added to cost functional (17a). These penalties drastically increase their values, whenever a constraint is violated or close to be violated. These characteristics force the optimal solution to satisfy the constraints. Consider a function $P_{z}$ defined as follows:

$P_{z}=P(z)= \begin{cases}(\underline{z}-z)^{n}, & z \leqslant \underline{z} \\ (z-\bar{z})^{n}, & z \geqslant \bar{z} \\ 0, & \text { otherwise. }\end{cases}$

with $\underline{z}$ the minimum allowed value for $z, \bar{z}$ the maximum allowed value for $z$, and $n>1$ a function parameter. $P_{z}$ is known as an exterior penalty function, as it only affects the cost functional when the constraints have been violated. Define $P_{u}$ and $P_{x}$ as the exterior penalty functions for constraints (17d) and (17e), respectively, and $\widetilde{P_{u}}=\phi \cdot P_{u}$, with $\phi=(1 / \bar{u})^{n}$. Given (17a), the penalty approach solves (17) by solving an equivalent unconstrained-OCP:

$\min \hat{J}(u)=\int_{0}^{t_{f}}\left[\vartheta \cdot \dot{m}_{f}(u)+d \cdot(1-\vartheta) \cdot u^{2}+\frac{1}{\varepsilon}\left(\widetilde{P_{u}}+P_{x}\right)\right] \mathrm{d} t$,

subject to:

(17b), (17c), (17f) - (17h),

where $\varepsilon$ is a real positive parameter pondering the penalty functions $\widetilde{P_{u}}$ and $P_{x}$. If $\varepsilon$ is small enough, the effect of $\widetilde{P_{u}}$ and $P_{x}$ increase to force the solution to (19), $\widehat{J}^{*}$, to be approximately equal to the solution of (17), $J^{*}$. Moreover, $\widehat{J}^{*} \rightarrow J^{*}$ as $\varepsilon \rightarrow 0$. Therefore, in order to get a fairly accurate approximation for the solution to (17), it is sufficient to solve (19) for a small enough value of $\varepsilon$. Proofs on the convergence of the penalty approach are given in [36,37].

\subsection{Optimality conditions}

Necessary optimality conditions for (19) can be obtained from the PMP. Before discussing the PMP necessary conditions, it is necessary to define the Hamiltonian of the OCP, denoted as $H$. The Hamiltonian for (19) is given by:

$H(u, x, \lambda \mid \vartheta, w)=\vartheta \cdot \dot{m}_{f}+d \cdot(1-\vartheta) \cdot u^{2}+\frac{1}{\varepsilon}\left(\widetilde{P_{u}}+P_{x}\right)+\lambda^{T} \dot{x}$

with $\lambda(t) \in \mathbb{R}^{2}$ denoting the vector of co-states and $x=\left[\begin{array}{ll}x_{1} & x_{2}\end{array}\right]^{T}$ denoting the vector of states. The necessary conditions of optimality for (19) are the following [38]: (i) the optimal control, $u^{*}(t)$, minimizes the 
Hamiltonian for every possible control:

$u^{*}(t)=\underset{u \in \mathbb{R}}{\operatorname{argmin}}\{H(u, x, \lambda \mid \vartheta, w)\}$,

(ii) the co-states obey the following dynamics:

$\dot{\lambda}_{1}(t)=-\frac{\partial H}{\partial x_{1}}$,

$\dot{\lambda}_{2}(t)=-\frac{\partial H}{\partial x_{2}}$,

and (iii) the following final value condition for $\lambda_{2}$ is met:

$\lambda_{2}(T)=0$.

It is not always possible to obtain $u^{*}$ explicitly. Nevertheless, let us assume by now that an explicit expression can be computed for $u^{*}$ and let us denote this expression as $\Pi(u, x, \lambda \mid \vartheta, w)$ :

$u^{*}(t)=\Pi(u, x, \lambda \mid \vartheta, w)$.

On the assumption that an optimal solution exists and that $H$ satisfies the following sufficient strict convexity condition with respect to $u$ :

$\frac{\partial^{2} H}{\partial u^{2}}>0$,

conditions (21)-(24) become necessary and sufficient optimality conditions $[39,40]$. Using (21)-(25), (19) can be formulated as an equivalent boundary value problem (BVP):

$\dot{x}_{1}(t)=\frac{1}{\bar{U} \cdot Q}\left[-(w-\vartheta \cdot \Pi)-\frac{R_{b}\left(x_{2}\right)}{U^{2}\left(x_{1}\right)}(w-\vartheta \cdot \Pi)^{2}\right]$,

$\dot{x}_{2}(t)=\frac{1}{C_{b}}\left[h \cdot\left(T_{\infty}-x_{2}\right)+\frac{R_{b}\left(x_{2}\right)}{U^{2}\left(x_{1}\right)}(w-\vartheta \cdot \Pi)^{2}\right]$,

$\dot{\lambda}_{1}(t)=-\frac{1}{\varepsilon} \frac{\partial P_{x}}{\partial x_{1}}$,

$\dot{\lambda}_{2}(t)=-\lambda_{1} \frac{\partial \dot{x}_{1}}{\partial x_{2}}-\lambda_{2} \frac{\partial \dot{x}_{2}}{\partial x_{2}}$,

$\lambda_{2}\left(t_{f}\right)=0$,

(17f) $-(17 \mathrm{~h})$.

This BVP can be solved through different numerical methods, e.g. shooting, multiple-shooting, or collocation $[41,42]$. Due to its numerical stability, a collocation method is applied in this paper [42]. It is worth noticing that the use of penalty functions increases the difficulty for minimizing the Hamiltonian, which is a necessary optimality condition. A solution to overcome this issue is presented in the following subsection.

\subsection{Implicit Hamiltonian minimization}

Firstly, consider the minimization of the Hamiltonian with respect to the input signal: optimality condition (21). For an unconstrained input signal and assuming that $H$ satisfies (26), the optimal control, $u^{*}(t)$, can be defined from the first order optimality conditions as follows:

$u^{*}(t)=\left\{u(t) \mid \frac{\partial H(u, x, \lambda \mid \vartheta, w)}{\partial u(t)}=0\right\}$

In general, (28) cannot be solved explicitly and an implicit Hamiltonian minimization can be used. Let us define $q(t)$ as follows: $q(t)=\frac{\partial H(u, x, \lambda \mid \vartheta, w)}{\partial u}$,

The following equivalent conditions for $u^{*}(t)$ can be obtained:

$q(0)=0$,

$\dot{q}(t)=0$,

From (31), it follows:

$\frac{\partial q}{\partial u} \dot{u}+\frac{\partial q}{\partial x_{1}} \dot{x}_{1}+\frac{\partial q}{\partial x_{2}} \dot{x}_{2}+\frac{\partial q}{\partial w} \dot{w}+\frac{\partial q}{\partial \vartheta} \dot{\vartheta}=0$,

from which the following optimal input dynamics can be computed:

$\dot{u}^{*}(t)=\left(\frac{\partial q}{\partial u}\right)^{-1}\left(\frac{\partial q}{\partial x_{1}} \dot{x}_{1}+\frac{\partial q}{\partial x_{2}} \dot{x}_{2}+\frac{\partial q}{\partial w} \dot{w}+\frac{\partial q}{\partial \vartheta} \dot{\vartheta}\right)$,

Therefore, the explicit minimization of the Hamiltonian can be replaced by (30) and (32), since $\frac{\partial q}{\partial u}=\frac{\partial^{2} H}{\partial u^{2}}$, (26) guarantees that $\left(\frac{\partial q}{\partial u}\right)^{-1}$ is defined. The methodology applied in order to approximate $\dot{w}$ and $\dot{\vartheta}$ is detailed in Appendix B. Considering these new optimality conditions, (19) can be solved via the following BVP [28]:

$\dot{x}_{1}(t)=\frac{1}{\bar{U} \cdot Q}\left[-\left(w-\vartheta \cdot u^{*}\right)-\frac{R_{b}\left(x_{2}\right)}{U^{2}\left(x_{1}\right)}\left(w-\vartheta \cdot u^{*}\right)^{2}\right]$,

$\dot{x}_{2}(t)=\frac{1}{C_{b}}\left[h \cdot\left(T_{\infty}-x_{2}\right)+\frac{R_{b}\left(x_{2}\right)}{U^{2}\left(x_{1}\right)}\left(w-\vartheta \cdot u^{*}\right)^{2}\right]$,

$\dot{\lambda}_{1}(t)=-\frac{1}{\varepsilon} \frac{\partial P_{x}}{\partial x_{1}}$,

$\dot{\lambda}_{2}(t)=-\lambda_{1} \frac{\partial \dot{x}_{1}}{\partial x_{2}}-\lambda_{2} \frac{\partial \dot{x}_{2}}{\partial x_{2}}$,

$\dot{u}^{*}(t)=\left(\frac{\partial q}{\partial u}\right)^{-1}\left(\frac{\partial q}{\partial x_{1}} \dot{x}_{1}+\frac{\partial q}{\partial x_{2}} \dot{x}_{2}+\frac{\partial q}{\partial w} \dot{w}+\frac{\partial q}{\partial \vartheta} \dot{\vartheta}\right)$,

$q(0)=0$,

(17f) $-(17 \mathrm{~h}),(27 f)$,

In the following, the solution to (33) will be denoted as $Y(t)=\left[x_{1}(t), x_{2}(t), \lambda_{1}(t), \lambda_{2}(t), u^{*}(t)\right]^{T}$. The dynamics of $Y$ will be denoted as $F(Y, t)$. The idea of computing higher order time derivatives of the Hamiltonian was also considered in [43]. There, an inversion approach that allows parameterizing the OCP using only one higherorder unknown parameter is presented. However, for the inversion method to be applied an explicit expression for the optimal control is required, which is not the case for the problem formulation considered in this paper.

\section{Numerical solution of the EMS}

When the state-of-energy reaches a boundary, the co-state is discontinuous [44]. BVP solvers cannot easily handle such discontinuities straightforwardly. This section deals with the numerical details that need to be considered when trying to solve BVP (33). The first two subsections discuss the continuation procedure, a numerical routine that breaks down the initial BVP into a sequence of several simpler subproblems, starting from an unconstrained one down to a fully constrained one. Solutions to these sub-problems may temporarily exceed the state and control limits and as a result, the solutions may lay outside the definition domain of $F(Y, t)$. To deal with this issue, the third subsection proposes to extend $F(Y, t)$ outside its domain of definition. 


\subsection{Continuation procedure}

The numerical success of the BVP solver depends on the quality of the provided initial guess solution. The difficulty of generating a good enough initial guess is overcome by the implementation of a continuation or homotopy procedure $[45,46]$. Before going into the application to the considered BVP, let us briefly recall how a continuation procedure is implemented in general.

Let us consider a BVP that depends on some parameters $\zeta$. The objective is to compute a solution for the parameter values $\zeta_{N}$ using a BVP solver. The continuation procedure, depicted in the Algorithm 1, requires an initial guess $Y_{0}$ derived for some given parameter values $\zeta_{0}$. At each iteration $i>0$ of the continuation procedure, the solution $Y_{i}$ of the BVP is numerically computed using $Y_{i-1}$ as an initial guess. The parameters are varied smoothly from their initial values $\zeta_{0}$ to their final values $\zeta_{N}$. The performance of the algorithm depends on the number of steps $N$ and the update function applied to vary the parameters from $\zeta_{0}$ to $\zeta_{N}$.

Algorithm 1. General continuation procedure $\lambda_{2}(0) \geqslant \frac{C_{b}}{U \cdot Q} \lambda_{1}(0)$. The corresponding initial optimal control $u(0)$ is numerically computed as a solution of $q(0)=0$. Given these initial conditions, a solution is then numerically computed by integrating (33a)-(33e) using an ODE solver. This solution is denoted by $Y^{a}(t)=\left[x_{1}^{a}(t), x_{2}^{a}(t), \lambda_{1}^{a}(t), \lambda_{2}^{a}(t), u^{* a}(t)\right]^{T}$.

\subsubsection{Step b: first continuation procedure}

The purpose of the first continuation procedure is to bring the final state-of-energy $x_{1}\left(t_{f}\right)$ to its prescribed final value $x_{1, f}$ and the second costate final value $\lambda_{2}\left(t_{f}\right)$ to zero. The penalty pondering coefficient $\varepsilon$ remains unchanged: $\varepsilon^{b}=\varepsilon^{a}$. The continuation procedure parameters are $\zeta^{b}=\left[x_{1}\left(t_{f}\right), \lambda_{2}\left(t_{f}\right)\right]^{T} . \quad \zeta_{0}^{b}$ is set equal to the final conditions $\left[x_{1}^{a}\left(t_{f}\right), \lambda_{2}^{a}\left(t_{f}\right)\right]^{T}$ from the initial solution $Y^{a} . \zeta_{N}^{b}$ is set to $\left[x_{1, f}, 0\right]^{T}$ as defined in (17h) and (27e). The parameter update function is assumed linear, values of $\zeta_{i}^{b}$ are given by:

$\zeta_{i}^{b}=\frac{\zeta_{N}^{b}-\zeta_{0}^{b}}{N^{b}}+\zeta_{i-1}^{b}$,

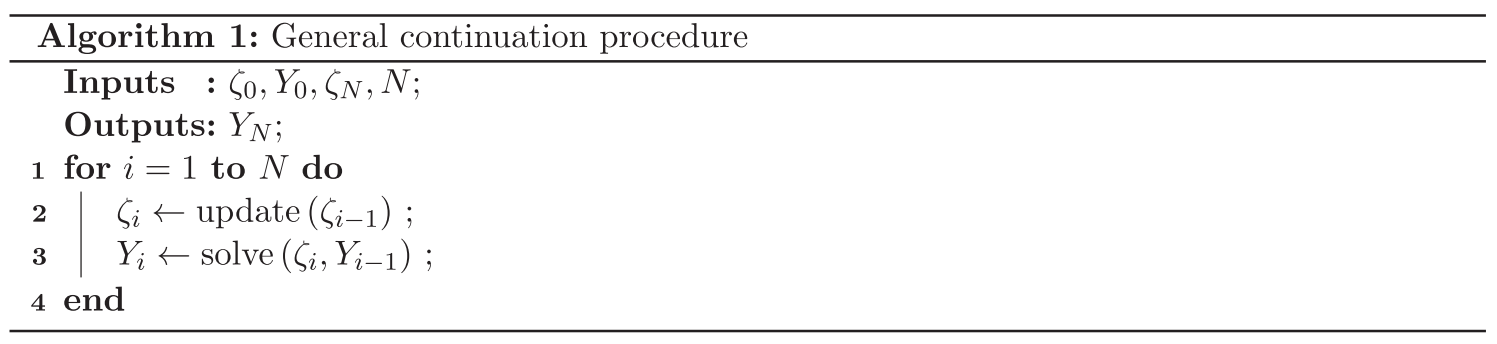

Algorithm 2. First continuation procedure.

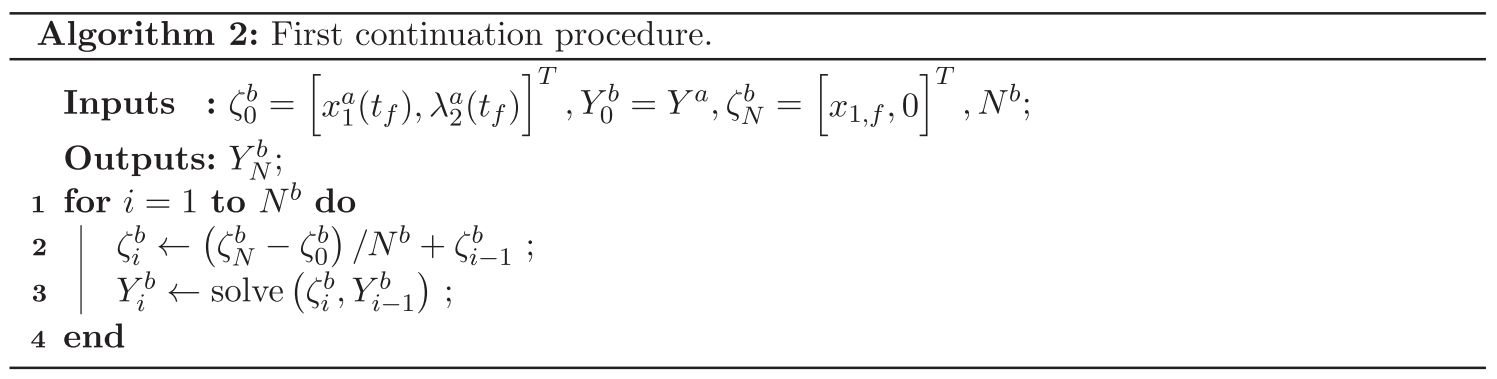

\subsection{Application to the considered problem}

In order to solve BVP (33), first, an initial guess is generated and then several continuation procedures are used. In order to differentiate the parameters of each continuation procedure, the following notation is introduced: $\zeta_{i}^{j}$ denotes the parameter values at iteration $i \in\{0,1, \ldots, N\}$ of the continuation procedure described in step $j \in\{a, b, c\}$.

\subsubsection{Step a: generation of an initial solution}

The penalty pondering coefficient $\varepsilon^{a}$ is initially set to a large value so the penalty functions are negligible. The initial co-states, $\left[\lambda_{1}(0), \lambda_{2}(0)\right]^{T}$ are selected such that assumption (26) is satisfied. Failing to meet this assumption could lead to $\frac{\partial^{2} H}{\partial u^{2}}=\frac{\partial q}{\partial u}$ equal to zero, rendering the optimal control, (33e), undefined. Given (20), (26) is satisfied if
The output of the continuation procedure is the unconstrained solution to (17). The continuation procedure is summarized in Algorithm 2.

\subsubsection{Step c: second continuation procedure}

The second continuation procedure activates the penalty functions by reducing the value of $\varepsilon$, thus $\zeta^{c}=\varepsilon$. $\zeta_{0}^{c}$ is set equal to the large value of $\varepsilon$ used initially: $\zeta_{0}^{c}=\varepsilon^{b}=\varepsilon^{a}$. In order to obtain a solution that fulfills the constraints, a very small value of $\varepsilon$ should be reached at the end of this continuation procedure: $\zeta_{N}^{c} \approx 0$. The values of $\zeta_{i}^{c}$ are chosen to be varied exponentially. The continuation procedure can be summarized in the following algorithm: 
Algorithm 3. Second continuation procedure.

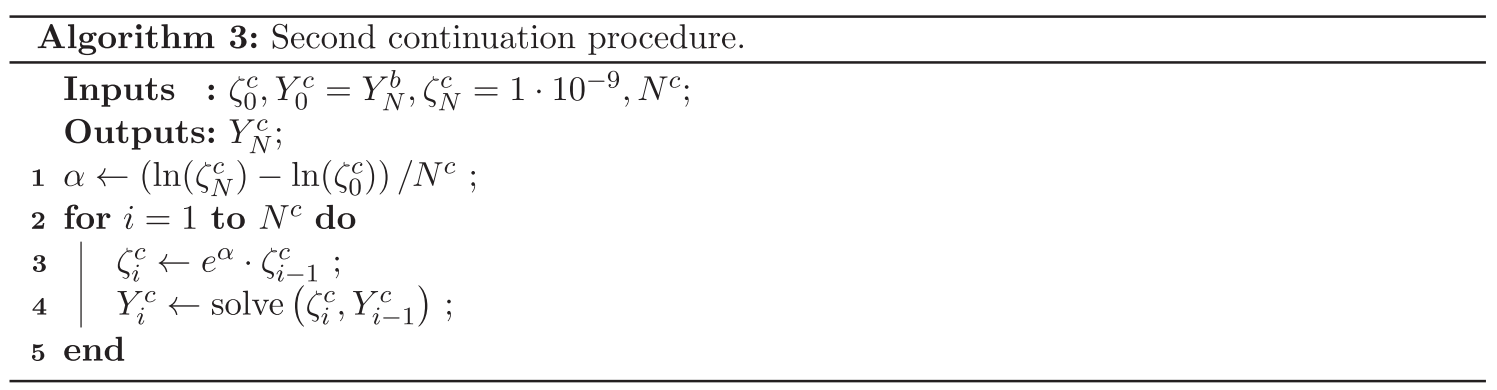

The output of the second continuation procedure is the solution to constrained BVP (33). The general numerical procedure presented in this subsection is summarized in Fig. 4.

\subsection{Function extension}

The dynamics $F(Y, t)$ is not defined for $U\left(x_{1}\right)=0$ due to the term $1 / U\left(x_{1}\right)$ in (8) and (11). As the only constraint for the initial co-states, used to generate the initial solution in Step a, is to satisfy (26), the states may reach values outside the definition domain of $F$. As a consequence, Step $a$ and $b$ may not be feasible. Moreover, even in Step $c$, the BVP solver may require to evaluate $F(Y, t)$ outside its definition domain. To overcome the latter problems, $F(Y, t)$ is extended outside its definition domain and the resulting function is denoted by $F_{\mathrm{ext}}(Y, t) \in \mathbb{R}^{5} . F$ and $F_{\text {ext }}$ are identical for all $Y \in Y_{\text {feas }} . F_{\text {ext }}$ is obtained by replacing $f\left(x_{1}\right)=1 / U\left(x_{1}\right):\left(-a_{x_{1}} / b_{x_{1}},+\infty\right] \rightarrow \mathbb{R}^{+}$by $f_{\text {ext }}\left(x_{1} \mid \widetilde{x_{1}}\right): \mathbb{R} \rightarrow \mathbb{R}^{+}$, with:

$f_{\text {ext }}\left(x_{1} \mid \widetilde{x_{1}}\right)= \begin{cases}1 / U\left(x_{1}\right), & \text { if } x_{1} \geqslant \widetilde{x_{1}} \\ g\left(x_{1}\right), & \text { otherwise }\end{cases}$

$$
\begin{aligned}
g\left(x_{1}\right)= & 1 / U\left(\widetilde{x_{1}}\right)+\left(x_{1}-\widetilde{x}_{1}\right) \frac{\mathrm{d}\left(1 / U\left(x_{1}\right)\right)}{\mathrm{d} x_{1}}+ \\
& \frac{1}{2}\left(x_{1}-\widetilde{x}_{1}\right)^{2} \frac{\mathrm{d}^{2}\left(1 / U\left(x_{1}\right)\right)}{\mathrm{d} x_{1}^{2}}
\end{aligned}
$$

where $\widetilde{x}_{1}$ is a scalar to be chosen from the interval $\left(-a_{x_{1}} / b_{x_{1}}, \underline{x}_{1}\right]$.

This extension is based on the Taylor series expansion and its generalization to terms depending on $n \in \mathbb{N}$ variables is given in Appendix A.

The Taylor-based function extension (35) and (36) preserves the convexity properties of the original function and thus guarantees that the Hamiltonian satisfies (26), which in turn guarantees that the solution remains unique and that (33e) is well defined. Choosing $\widetilde{x}_{1}=\underline{x}_{1}=0.1,1 / U\left(x_{1}\right)$ and $f_{\text {ext }}\left(x_{1} \mid \widetilde{x}_{1}\right)$ are shown in Fig. 5 along with $x_{1}=-a_{x_{1}} / b_{x_{1}}$, the limit of the definition domain for $1 / U\left(x_{1}\right)$.

\section{Numerical results}

Considering the vehicle parameters contained in Table 1 , the offline-EMS is solved under low-temperature and warm operation conditions in order to study the effect of the battery temperature on the fuel consumption.

\subsection{Low-temperature operation}

The low-temperature operation is defined here as starting the vehicle with $T_{\infty}=253.15 \mathrm{~K}\left(-20{ }^{\circ} \mathrm{C}\right)$, where $T_{\infty}$ is assumed to remain constant along the entire driving cycle. At the beginning of the driving cycle, the following assumption holds: $x_{2}=T_{\infty}$. Considering the WLTC-C3 driving cycle, the optimal offline-EMS defined in (17) is solved via the equivalent BVP (33) using a collocation solver [42] and the numerical procedure and function extension described in Section 4.

First, the initial guess $Y^{a}$ is generated as described in Step a using $\lambda(0)=\left[\begin{array}{ll}1.4 \cdot 10^{5} & 1.45 \cdot 10^{5}\end{array}\right]^{T}$ and the initial penalty pondering coefficient $\varepsilon^{a}=1 \cdot 10^{12}$. The obtained initial solution $Y^{a}$ is depicted in Fig. 6 . The state-of-energy reaches negative values, showing that the initial guess does not necessarily have a physical meaning.

The final conditions from $Y^{a},\left[x_{1}^{a}\left(t_{f}\right), \lambda_{2}^{a}\left(t_{f}\right)\right]^{T}=\left[-7.3,5.32 \cdot 10^{6}\right]^{T}$, are applied at the beginning of Step $b: \zeta_{0}^{b}=\left[x_{1}^{a}\left(t_{f}\right), \lambda_{2}^{a}\left(t_{f}\right)\right]^{T}$. Step $b$ allows computing a solution that reaches the expected final state-of-energy and the necessary final condition for the second co-state: $\zeta_{f}^{b}=\left[x_{1}\left(t_{f}\right), 0\right]^{T}$. Its output $Y_{N}^{b}$, shown in Fig. 7, is the solution to EMS (17) without constraints, since the penalty functions are negligible: $\varepsilon^{b}=\varepsilon^{a}=1 \cdot 10^{12}$.

The output of Step $b, Y_{N}^{b}$, is fed into Step $c$ : $Y_{0}^{c}=Y_{N}^{b}$. Step $c$ is used to activate the penalty functions. The initial penalty pondering coefficient is set to the same value of Step $b: \zeta_{0}^{c}=\varepsilon_{0}^{c}=\varepsilon^{b}=1 \cdot 10^{12}$. The final penalty pondering coefficient is set to a small positive value: $\varepsilon_{N}^{c}=1 \cdot 10^{-9}$. The output of Step c, $Y_{N}^{c}$, is the solution to constrained EMS (17) and is shown in Fig. 8. It allows computing the fuel consumption for the lowtemperature operation: $9.85 \mathrm{~L} / 100 \mathrm{~km}$.

The constrained solution for the EMS, Fig. 8, shows a discontinuity

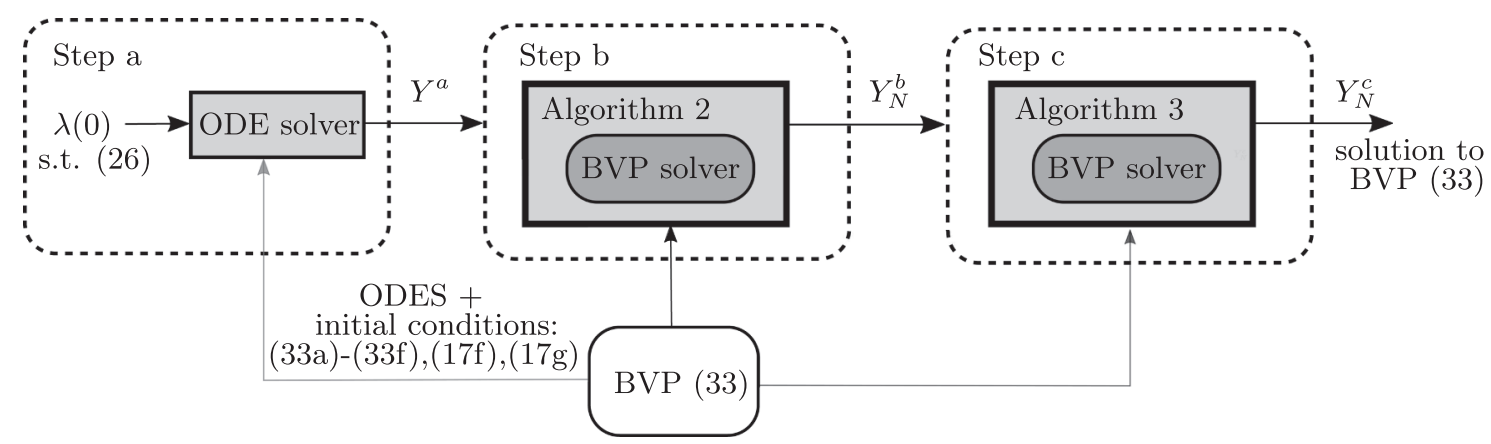

Fig. 4. Overview of Step a, Step b, and Step c. 


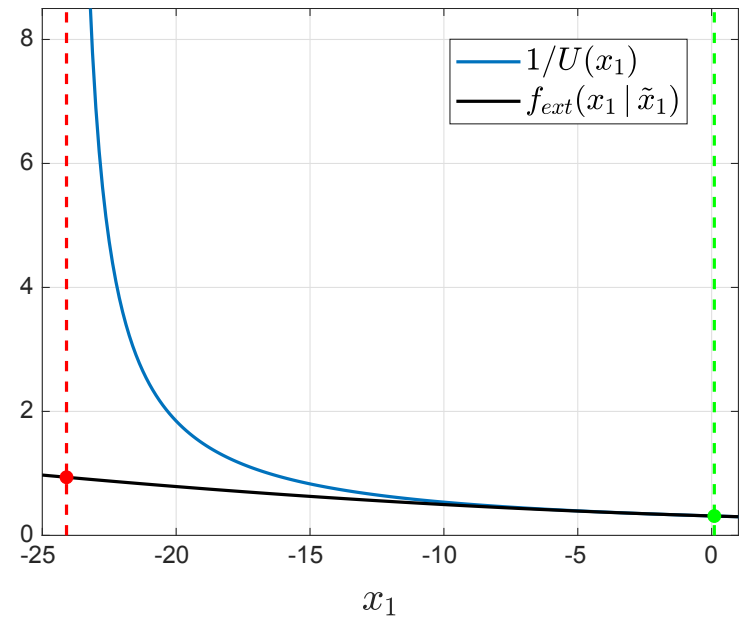

Fig. 5. $1 / U\left(x_{1}\right)$ (blue line), its function extension $f_{\text {ext }}\left(x_{1} \mid \widetilde{x}_{1}\right)$ (black line), the limit of the definition domain of $1 / U\left(x_{1}\right): x_{1}=-a_{x_{1}} / b_{x_{1}}$ (red dotted line), and $\widetilde{x}_{1}=\underline{x}_{1}=0.1$ (green dotted line).

Table 1

Parameters considered in the numerical experiments.

\begin{tabular}{ccc}
\hline Parameter & Value & Units \\
\hline$M_{e q}$ & 2166 & $\mathrm{~kg}$ \\
$A_{f}$ & 1.98 & $\mathrm{~m}^{2}$ \\
$c_{d}$ & 0.32 & - \\
$c_{r}$ & 0.01 & - \\
$\gamma$ & 4.2 & - \\
$r_{w}$ & 0.26 & $\mathrm{~m}$ \\
$g$ & 9.81 & $\mathrm{~kg} \cdot \mathrm{s}^{2}$ \\
$J_{t m}$ & 0.045 & $\mathrm{~kg} \cdot \mathrm{m}^{2}$ \\
$I$ & -40 & $\mathrm{~A}$ \\
$\bar{I}$ & 40 & $\mathrm{~A}$ \\
$a_{x_{1}}$ & 653.85 & $\mathrm{~V}$ \\
$b_{x_{1}}$ & 27.14 & $\mathrm{~V}$ \\
$Q$ & 1800 & $\mathrm{~A} \cdot \mathrm{s}$ \\
$h$ & 4.343 & $\mathrm{~W} / \mathrm{K}$ \\
$C_{b}$ & 142.56 & $\mathrm{~kJ} / \mathrm{K}$ \\
$a$ & 0.2924 & $\mathrm{~g} / \mathrm{s}$ \\
$b$ & 0.0834 & $\frac{\mathrm{g}}{\mathrm{kW} \cdot \mathrm{s}}$ \\
$c$ & 0.0055 & $\frac{\mathrm{g}}{(\mathrm{kW})^{2} \cdot \mathrm{s}}$ \\
$d$ & 1 & $\mathrm{~g}$ \\
$\bar{u}$ & 17.5 & $\mathrm{~kW})^{2} \cdot \mathrm{s}$ \\
& & $\mathrm{kW}$ \\
\hline & & \\
\hline & &
\end{tabular}

phenomenon for $\lambda_{1}(t)$. This phenomenon is to be expected, whenever a state makes contact with its bounds [44]. It is worth noticing that the procedure proposed in this work does not require any a priori knowledge about these discontinuities.

\subsection{Warm versus low-temperature operation}

Here, the fuel consumption of the low-temperature operation will be compared with an ideal warm operation. The warm operation is defined as $T_{\infty}=298.15 \mathrm{~K}\left(25^{\circ} \mathrm{C}\right)$, with $T_{\infty}$ assumed constant along the entire driving cycle. At the beginning of the driving cycle $x_{2}=T_{\infty}$ holds. Moreover, in the warm operation, the dynamics of the battery temperature is considered to be equal to zero; on the assumption that a cooling system is in place to keep $x_{2} \approx 298.15 \mathrm{~K}\left(25^{\circ} \mathrm{C}\right)$ along the entire driving cycle. The offline-EMS is solved under warm operation
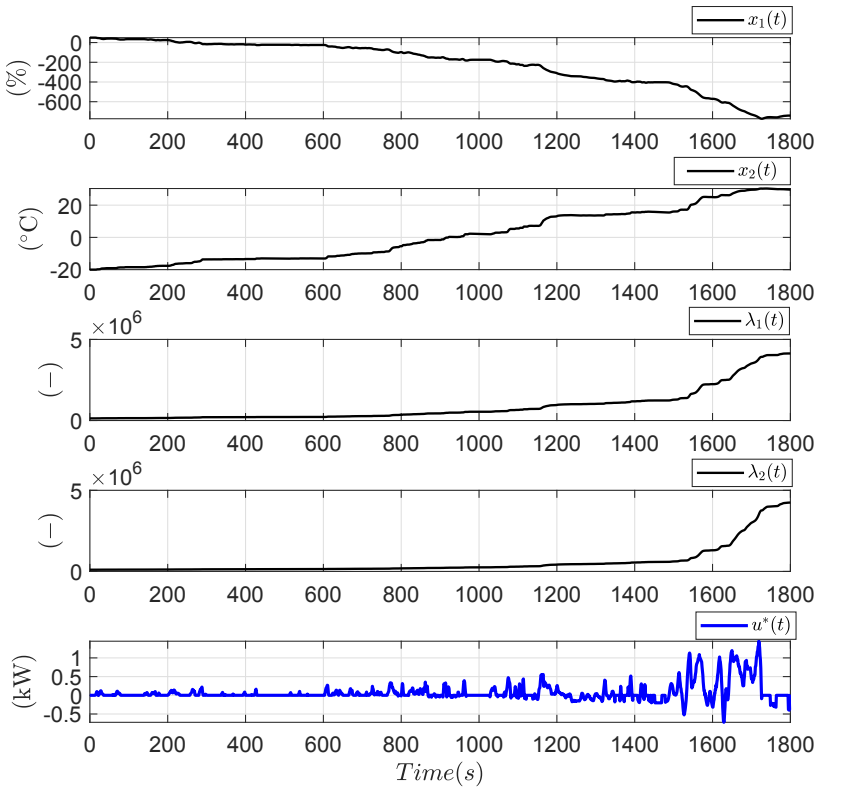

Fig. 6. Initial guess $Y^{a}$ generated in Step a for the WLTC-C3 driving cycle.
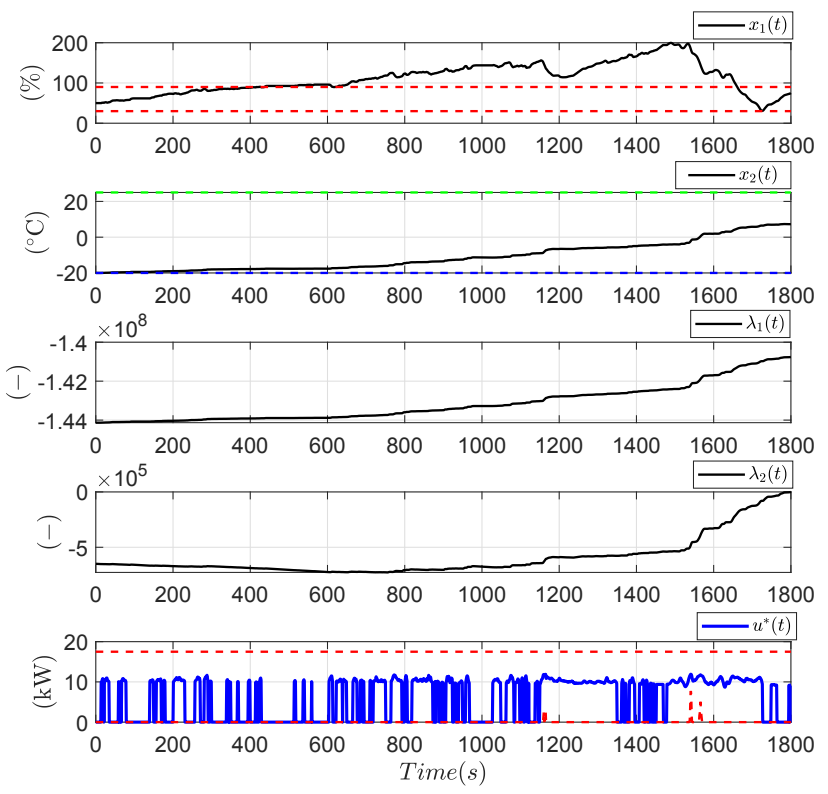

Fig. 7. Unconstrained solution to (17) for the low-temperature operation and the WLTC-C3 driving cycle. The fuel consumption is $9.53 \mathrm{~L} / 100 \mathrm{~km}$. The red dashed lines at the top and bottom subfigures represent the bounds on the state-ofenergy and the control input, (17e) and (17d), respectively.

conditions using the same vehicle parameters of the low-temperature operation. The fuel consumption for each operation is shown in Table 2. The low-temperature operation increases the fuel consumption with respect to the ideal warm operation in a $4.01 \%$.

\subsection{Comparison with $D P$}

The optimal energy management for the low-temperature operation will be solved again using DP. The objective is to validate the optimality 


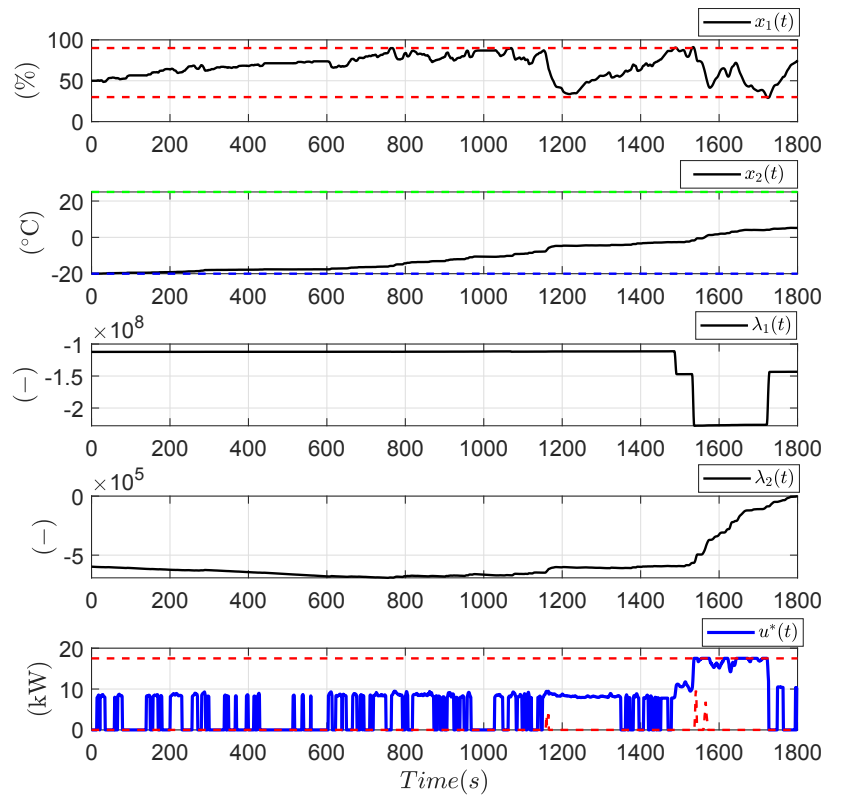

Fig. 8. Constrained solution to (17) for the low-temperature operation and the WLTC-C3 driving cycle. The fuel consumption is $9.85 \mathrm{~L} / 100 \mathrm{~km}$. The red dashed lines at the top and bottom subfigures represent the bounds on the state-ofenergy and the control input, (17e) and (17d), respectively.

Table 2

Fuel consumption results for each operating condition.

\begin{tabular}{ccc}
\hline Operation & Fuel consumption (WLTC-C3) & Difference \\
\hline Warm & $9.47 \mathrm{~L} / 100 \mathrm{~km}$ & - \\
Low-temperature & $9.85 \mathrm{~L} / 100 \mathrm{~km}$ & $+4.01 \%$ \\
\hline
\end{tabular}

of the proposed approach and to benchmark its computational efficiency. As mentioned in Section 1, DP has been widely used to solve the EMS in the literature of hybrid electric vehicles $[11,12]$. Its advantages are that it can solve the EMS with mixed input-state constraints with a guarantee of global optimality. Its main drawback is that it has an exponential growth of memory and computational complexity with respect to the number of states, denoted as the curse of dimensionality [10]. For this reason, its use is limited to EMS formulations with one [11] or two states [13]. The DP algorithm is implemented using the Matlab code from [47], together with the iterative approach described in [48]. The results of the DP solution to (17) are displayed in Fig. 9 along with the solution computed with the proposed approach. The fuel consumption and computation times are compared in Table 3. The proposed approach is 46 times faster than DP but obtains $+0.4 \%$ more fuel consumption as well. The small difference in fuel consumption can be due to the limited accuracy that DP posses as a consequence of the quantization of all variables and the Euler integration scheme in which it relies on.

\section{Conclusion}

An indirect-approach method to compute the optimal offline energy management strategy of a hybrid vehicle with several continuous states
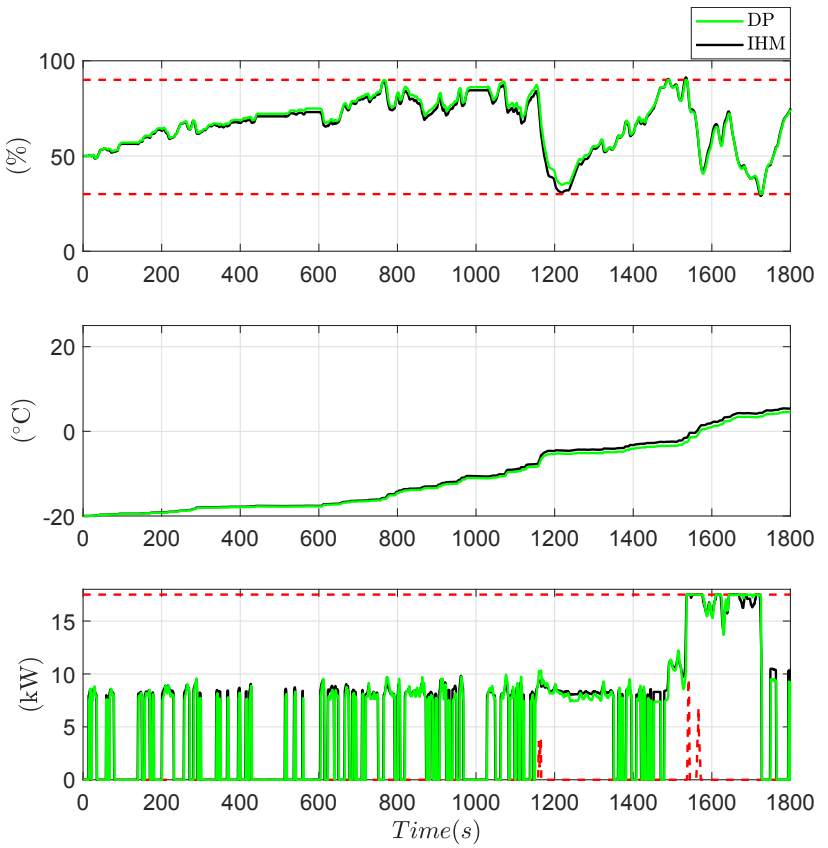

Fig. 9. Solution to (17) for the low-temperature operation and the WLTC-C3 driving cycle with DP (green solid line) and the proposed implicit Hamiltonian minimization (black solid line). The red dashed lines at the top and bottom subfigures represent the bounds on the state-of-energy and the control input, (17e) and (17d), respectively.

and under input and state constraints has been proposed. The indirectapproach method relies on exterior penalty functions and an implicit Hamiltonian minimization to handle the constraints and satisfy the Pontryagin's minimum principle optimality conditions. A continuation procedure and a domain function-extension are also proposed to deal with the difficulties of computing a numerical solution. The proposed method does not require any a priori knowledge on the optimal solution and can be applied to energy management formulations with several inputs and states.

The proposed method is shown effective by solving the offline energy management strategy of a hybrid vehicle under low-temperature conditions modeled as a two-state, the battery state-of-energy and temperature, mixed input-state constrained problem. The optimality of the solution is validated by obtaining an slightly different solution via dynamic programming. The small difference is not surprising since in dynamic programming all variables are quantized, which limits the accuracy of the solution. Moreover, the proposed method shows to be up to 46 times faster than dynamic programming.

One possible extension of this work is to solve a more general energy management formulation, for example, by considering both the engine and the catalytic converter temperature and including the emission of pollutants in the objective function $[2,49]$. Also, real-time algorithms for hybrid vehicle energy management have been derived using the Pontryagin's minimum principle within a model predictive control framework [50]. The proposed method would allow to account for both mixed input-state constraints and a second dynamics in realtime but more work is needed to cope with the actual computing power available on the vehicle. 
Table 3

Fuel consumption and computation time for DP and the proposed implicit Hamiltonian minimization under low-temperature conditions.

\begin{tabular}{ccc}
\hline Method & Fuel DP (WLTC-C3) & Computation time (s) \\
\hline DP & $9.81 \mathrm{~L} / 100 \mathrm{~km}$ & $6.187 \cdot 10^{4}$ \\
Implicit Hamiltonian minimization & $9.85 \mathrm{~L} / 100 \mathrm{~km}$ & $1.319 \cdot 10^{3}$ \\
\hline
\end{tabular}

\section{Acknowledgment}

The present research was supported by the Regional Delegation for Research and Technology, the French Ministry of Higher Education and Research, and the National Center for Scientific Research. It was also supported by the ELSAT2020 project, co-funded by the European Regional Development Fund, the French state and the Hauts de France Region Council. The authors greatly acknowledge these institutions for their support.

\section{Appendix A. Function extension with multiple variables}

Let us consider $f_{i} \in X_{i} \subset \mathbb{R}^{n_{i}}$, where $X_{i}$ is a convex region and $f_{i}$ is $C^{\infty}$. The Taylor series expansion of $f_{i}$ around of $\tilde{x}$ is defined as follows:

$$
\begin{aligned}
f_{i}(x)= & f_{i}(\widetilde{x})+(x-\widetilde{x})^{T} \nabla f_{i}(\widetilde{x})+ \\
& +\frac{1}{2}(x-\widetilde{x})^{T} \nabla^{2} f_{i}(\widetilde{x})(x-\widetilde{x})+\ldots
\end{aligned}
$$

With $\nabla f_{i}$ and $\nabla^{2} f_{i}$ defined as the gradient and Hessian matrix of $f_{i}$, respectively. Given $\widetilde{x} \in \partial X_{i}$, and $y \notin X$, the domain of $f_{i}$ is extended outside $X_{i}$ by the following function:

$g_{i}(y)=f_{i}(\widetilde{x})+(y-\widetilde{x})^{T} \nabla f_{i}(\widetilde{x})+\frac{1}{2}(y-\widetilde{x})^{T} \nabla^{2} f_{i}(\widetilde{x})(y-\widetilde{x})$,

where $\tilde{x}$ is defined as the closest point on the boundary of $X_{i}, \partial X_{i}$, with respect to $y$.

\section{Appendix B. Piecewise polynomial approximation of the power demand and the On/Off signal command}

The time derivatives of the power demand, $\dot{w}(t)$, and the On/Off command of the APU, $\dot{\vartheta}(t)$, are necessary to compute the optimal control dynamics $\dot{u}^{*}(t)$, see (33e). Since these signals are not available, a monotone piecewise cubic interpolation [51] is applied to $w(t)$ and $\vartheta(t)$ in order to approximate them. The interpolating polynomials of $w(t)$ and $\vartheta(t)$, denoted as $p_{w}(t)$ and $p_{\vartheta}(t)$, respectively, are guaranteed to be at least once continuously differential. The difference between the signals and its piecewise cubic approximations can be arbitrarily reduced by increasing the number of interpolating points at the cost of a greater computational effort.

The computation of the interpolating polynomials is carried out using a predefined Matlab function: pchip. Once $p_{w}(t)$ and $p_{\vartheta}(t)$ have been computed, $\dot{p}_{w}(t)$ and $\dot{p}_{\vartheta}(t)$ are used to approximate $\dot{w}(t)$ and $\dot{\vartheta}(t)$, respectively.

\section{Appendix C. Efficiency and BSFC maps}

The APU model computed using a static efficiency map for the generator and a BSFC map for the engine [35,52], see Fig. C.10. The traction motor is modeled using a static efficiency map $\eta_{t m}\left(T_{t m}, \omega_{t m}\right)$, see Fig. C.11.

Fuel consumption model (12) is computed using a quadratic fitting for a set of optimal pairs $\left\{\dot{m}_{f}^{k}\left(T_{i c e}^{k}, \omega_{i c e}^{k}\right), u^{k}\right\}$, where $T_{i c e}^{k}$ and $\omega_{i c e}^{k}$ are the ICE operating conditions necessary to generate $u^{k}$ electric power with minimum fuel consumption. The optimal pairs correspond to the APU operating conditions shown in Fig. C.10. $\dot{m}_{f}^{k}$ is computed by solving the following optimization problem for each $u^{k}$ :

$\min _{T_{i c e}, \omega_{i c e}} \dot{m}_{f}\left(T_{i c e}, \omega_{i c e}\right)$,
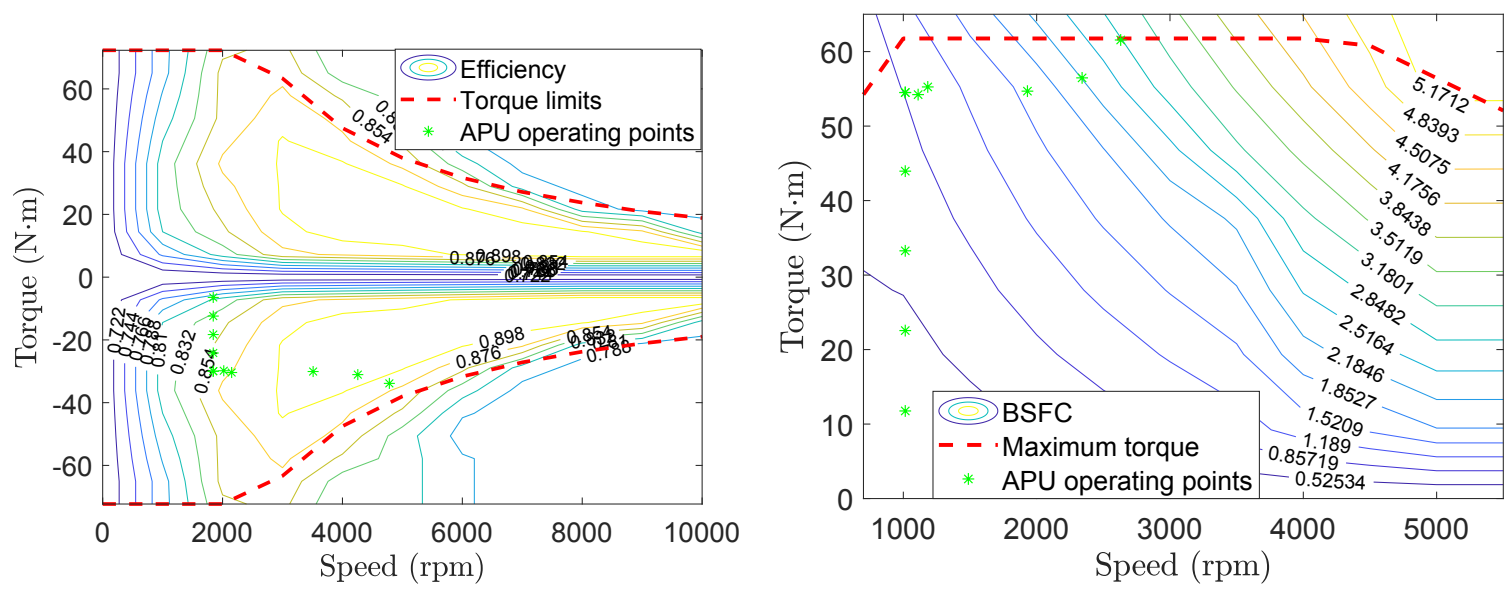

Fig. C.10. Efficiency map of the generator (left). Brake specific fuel consumption (BSFC) map ( $\mathrm{g} / \mathrm{s}$ ) of the ICE (right). 


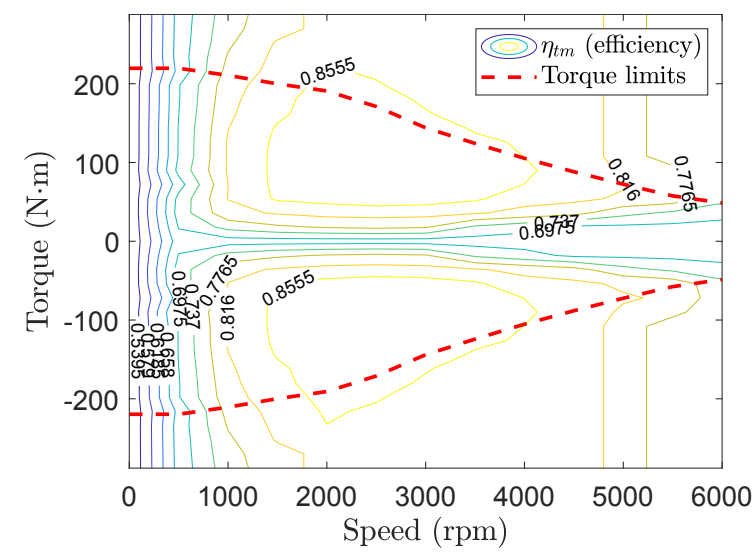

Fig. C.11. Efficiency map of the traction motor $\eta_{t m}$.

subject to:

$\eta_{\text {gen }}\left(T_{\text {gen }}, \omega_{\text {gen }}\right) \cdot T_{\text {gen }} \cdot \omega_{\text {gen }}=u^{k}$,

$T_{i c e}=T_{\text {gen }} \cdot \rho$,

$\omega_{\text {ice }}=\frac{\omega_{i c e}}{\rho}$,

$\underline{\omega}_{\text {gen }} \geqslant \omega_{\text {gen }}(t) \geqslant \bar{\omega}_{\text {gen }}$,

$\underline{\omega}_{i c e} \geqslant \omega_{\text {ice }}(t) \geqslant \bar{\omega}_{\text {ice }}$,

$\underline{T}_{\text {gen }}\left(\omega_{\text {gen }}(t)\right) \geqslant T_{\text {gen }}(t) \geqslant \bar{T}_{\text {gen }}\left(\omega_{\text {gen }}(t)\right)$,

$\underline{T}_{i c e}\left(\omega_{i c e}(t)\right) \geqslant T_{i c e}(t) \geqslant \bar{T}_{i c e}\left(\omega_{i c e}(t)\right)$,

with $T_{\text {gen }}$ and $\omega_{\text {gen }}$ the torque and speed of the generator, respectively, and $\rho$ the gear ratio between the ICE and the generator.

\section{References}

[1] Guzzella L, Sciarretta A. Vehicle propulsion systems: introduction to modeling and optimization. Springer Science \& Business Media; 2012.

[2] Kum D, Peng H, Bucknor NK. Optimal energy and catalyst temperature management of plug-in hybrid electric vehicles for minimum fuel consumption and tail-pipe emissions. IEEE Trans Control Syst Technol 2013;21(1):14-26.

[3] Michel P, Charlet A, Colin G, Chamaillard Y, Bloch G, Nouillant C. Optimizing fuel consumption and pollutant emissions of gasoline-HEV with catalytic converter. Control Eng Pract 2017;61:198-205.

[4] Opila DF, Wang X, McGee R, Gillespie RB, Cook JA, Grizzle JW. An energy management controller to optimally trade off fuel economy and drivability for hybrid vehicles. IEEE Trans Control Syst Technol 2012;20(6):1490-505.

[5] Miro-Padovani T, Colin G, Ketfi-Chérif A, Chamaillard Y. Implementation of an energy management strategy for hybrid electric vehicles including drivability constraints. IEEE Trans Veh Technol 2016;65(8):5918-29.

[6] Ebbesen S, Elbert P, Guzzella L. Battery state-of-health perceptive energy management for hybrid electric vehicles. IEEE Trans Veh Technol 2012;61(7):2893-900.

[7] Sciarretta A, di Domenico D, Pognant-Gros P, Zito G. "Optimal energy management of automotive battery systems including thermal dynamics and aging. Optimization and optimal control in automotive systems. Springer; 2014. p. 219-36.

[8] Hou C, Ouyang M, Xu L, Wang H. Approximate Pontryagin's minimum principle applied to the energy management of plug-in hybrid electric vehicles. Appl Energy 2014;115:174-89.

[9] Silvas E, Hofman T, Murgovski N, Etman LP, Steinbuch M. Review of optimization strategies for system-level design in hybrid electric vehicles. IEEE Trans Veh Technol 2016;66(1):57-70.

[10] Bellman RE. Dynamic programming. Courier Dover Publications; 1957.

[11] Lin C-C, Peng H, Grizzle JW, Kang J-M. Power management strategy for a parallel hybrid electric truck. IEEE Trans Control Syst Technol 2003;11(6):839-49.

[12] Ebbesen S, Dönitz C, Guzzella L. Particle swarm optimisation for hybrid electric drive-train sizing. Int J Veh Des 2012;58(2-4):181-99.

[13] Ansarey M, Panahi MS, Ziarati H, Mahjoob M. Optimal energy management in a dual-storage fuel-cell hybrid vehicle using multi-dimensional dynamic programming. J Power Sources 2014;250:359-71.

[14] Yang Y, Hu X, Pei H, Peng Z. Comparison of power-split and parallel hybrid powertrain architectures with a single electric machine: dynamic programming approach. Appl Energy 2016;168:683-90.

[15] Zhu Y, Chen Y, Tian G, Wu H, Chen Q. A four-step method to design an energy management strategy for hybrid vehicles. In: Proceedings of the 2004 American control conference, vol. 1. IEEE; 2004. p. 156-61.

[16] Khalik Z, Padilla G, Romijn T, Donkers M. Vehicle energy management with ecodriving: a sequential quadratic programming approach with dual decomposition. In: 2018 Annual American control conference (ACC). IEEE; 2018. p. 4002-7.

[17] Pourabdollah M, Silvas E, Murgovski N, Steinbuch M, Egardt B. Optimal sizing of a series PHEV: comparison between convex optimization and particle swarm optimization. IFAC-PapersOnLine 2015;48(15):16-22.

[18] Romijn TCJ, Donkers M, Kessels JT, Weiland S. A distributed optimization approach for complete vehicle energy management. IEEE Trans Control Syst Technol 2018;99:1-17.

[19] Tate ED, Boyd SP. Finding ultimate limits of performance for hybrid electric vehicles. SAE Technical Paper. Tech. Rep.; 2000.

[20] Zulkefli MAM, Sun Z. Fast numerical powertrain optimization strategy for connected hybrid electric vehicles. IEEE Trans Veh Technol 2019.

[21] Pourabdollah M, Murgovski N, Grauers A, Egardt B. Optimal sizing of a parallel PHEV powertrain. IEEE Trans Veh Technol 2013;62(6):2469-80.

[22] Riedinger P, Iung C, Kratz F. An optimal control approach for hybrid systems. Eur J Control 2003;9(5):449-58.

[23] Ngo V, Hofman T, Steinbuch M, Serrarens A. Optimal control of the gearshift command for hybrid electric vehicles. IEEE Trans Veh Technol 2012;61(8):3531-43.

[24] Delprat S, Hofman T, Paganelli S. Hybrid vehicle energy management: singular optimal control. IEEE Trans Veh Technol 2017;66(11):9654-66.

[25] van Keulen T, Gillot J, de Jager B, Steinbuch M. Solution for state constrained optimal control problems applied to power split control for hybrid vehicles. Automatica 2014;50(1):187-92.

[26] Kareemulla T, Delprat S, Czelecz L. State constrained hybrid vehicle optimal energy management: an interior penalty approach. IFAC-PapersOnLine, vol. 50, no. 1; 2017. p. 10040-5.

[27] Han J, Sciarretta A, Petit N. Handling state constraints in fast-computing optimal control for hybrid powertrains. IFAC-PapersOnLine 2017;50(1):4781-6.

[28] Sanchez M, Delprat S. Hybrid vehicle energy management: avoiding the explicit hamiltonian minimization. In: 2018 IEEE vehicle power and propulsion conference (VPPC). IEEE; 2018. p. 1-5.

[29] Wang C, Yang R, Yu Q. Wavelet transform based energy management strategies for plug-in hybrid electric vehicles considering temperature uncertainty. Appl Energy 2019;256:113928.

[30] Ruan J, Walker PD, Watterson PA, Zhang N. The dynamic performance and economic benefit of a blended braking system in a multi-speed battery electric vehicle. 
Appl Energy 2016;183:1240-58.

[31] Padovani TM, Debert M, Colin G, Chamaillard Y. Optimal energy management strategy including battery health through thermal management for hybrid vehicles. IFAC Proc Vol 2013;46(21):384-9.

[32] Jaguemont J, Boulon L, Dubé Y. Characterization and modeling of a hybrid-electricvehicle lithium-ion battery pack at low temperatures. IEEE Trans Veh Technol 2016;65(1):1-14.

[33] Lin X, Perez HE, Siegel JB, Stefanopoulou AG, Li Y, Anderson RD, et al. Online parameterization of lumped thermal dynamics in cylindrical lithium ion batterie for core temperature estimation and health monitoring. IEEE Trans Control Syst Technol 2013;21(5):1745-55.

[34] Yang Z, Patil D, Fahimi B. Electrothermal modeling of lithium-ion batteries for electric vehicles. IEEE Trans Veh Technol 2019;68(1):170-9.

[35] Murgovski N, Johannesson L, Hellgren J, Egardt B, Sjöberg J. Convex optimization of charging infrastructure design and component sizing of a plug-in series hev powertrain. IFAC Proc Vol 2011;44(1):13052-7.

[36] Lasdon L, Waren A, Rice R. An interior penalty method for inequality constrained optimal control problems. IEEE Trans Autom Control 1967;12(4):388-95.

[37] Lele MM, Jacobson DH. A proof of the convergence of the Kelley-Bryson penalty function technique for state-constrained control problems. J Math Anal Appl 1969;26(1):163-9.

[38] Kirk DE. Optimal control theory: an introduction. Courier Corporation; 2012.

[39] Naidu DS. Optimal control systems. CRC Press; 2002.

[40] Siouris GM. An engineering approach to optimal control and estimation theory. John Wiley \& Sons, Inc; 1996.

[41] Ascher UM, Mattheij RM, Russell RD. Numerical solution of boundary value problems for ordinary differential equations. Siam 1994;13.

[42] Kierzenka J, Shampine LF. A BVP solver based on residual control and the Maltab PSE. ACM Trans Math Softw 2001;27(3):299-316.

[43] Petit N, Sciarretta A. Optimal drive of electric vehicles using an inversion-based trajectory generation approach. IFAC Proc Vol 2011;44(1):14519-26.

[44] Bonnans JF, Hermant A. Second-order analysis for optimal control problems with pure state constraints and mixed control-state constraints. In: Annales de l'Institut Henri Poincare (C) Non Linear Analysis, vol. 26, no. 2. Elsevier; 2009. p. 561-98.

[45] Fabien BC. Numerical solution of constrained optimal control problems with parameters. Appl Math Comput 1996;80(1):43-62.

[46] Graichen K, Petit N. Constructive methods for initialization and handling mixed state-input constraints in optimal control. J Guid Control Dyn 2008;31(5):1334-43.

[47] Sundstrom O, Guzzella L. A generic dynamic programming matlab function. In: 2009 IEEE control applications, (CCA) \& intelligent control, (ISIC). IEEE; 2009. pp 1625-30.

[48] Luus R. Iterative dynamic programming. Chapman and Hall/CRC; 2000.

[49] Merz F, Sciarretta A, Dabadie J-C, Serrao L. On the optimal thermal management of hybrid-electric vehicles with heat recovery systems. Oil Gas Sci Technol-Revue d'IFP Energies nouvelles 2012;67(4):601-12.

[50] Xie S, Hu X, Xin Z, Brighton J. Pontryagin's minimum principle based model predictive control of energy management for a plug-in hybrid electric bus. Appl Energy 2019;236:893-905.

[51] Fritsch FN, Carlson RE. Monotone piecewise cubic interpolation. SIAM J Numer Anal 1980;17(2):238-46.

[52] Chen Z, Xia B, You C, Mi CC. A novel energy management method for series plug-in hybrid electric vehicles. Appl Energy 2015;145:172-9. 